\title{
Full-F gyrofluid model
}

\section{Madsen, Jens}

\section{Published in:}

Physics of Plasmas

Link to article, DOI:

$10.1063 / 1.4813241$

Publication date:

2013

Document Version

Publisher's PDF, also known as Version of record

Link back to DTU Orbit

Citation (APA):

Madsen, J. (2013). Full-F gyrofluid model. Physics of Plasmas, 20(7), 072301. https://doi.org/10.1063/1.4813241

\section{General rights}

Copyright and moral rights for the publications made accessible in the public portal are retained by the authors and/or other copyright owners and it is a condition of accessing publications that users recognise and abide by the legal requirements associated with these rights.

- Users may download and print one copy of any publication from the public portal for the purpose of private study or research.

- You may not further distribute the material or use it for any profit-making activity or commercial gain

- You may freely distribute the URL identifying the publication in the public portal

If you believe that this document breaches copyright please contact us providing details, and we will remove access to the work immediately and investigate your claim. 


\section{$\mathrm{AIP} \mid \begin{aligned} & \text { Physics of } \\ & \text { Plasmas }\end{aligned}$}

\section{Full-F gyrofluid model}

\section{Jens Madsen}

Citation: Physics of Plasmas (1994-present) 20, 072301 (2013); doi: 10.1063/1.4813241

View online: http://dx.doi.org/10.1063/1.4813241

View Table of Contents: http://scitation.aip.org/content/aip/journal/pop/20/7?ver=pdfcov

Published by the AIP Publishing

AAlP Re-register for Table of Content Alerts

Create a profile.

Sign up today! 


\title{
Full-F gyrofluid model
}

\author{
Jens Madsen ${ }^{\text {a) }}$ \\ Department of Physics, Association EURATOM-DTU, Technical University of Denmark, DK-4000 Roskilde, \\ Denmark
}

(Received 4 December 2012; accepted 3 June 2013; published online 10 July 2013)

\begin{abstract}
A global electromagnetic gyrofluid model based on the full-F gyrokinetic model is derived. The gyrofluid moment variables are not split into fluctuating and equilibrium parts. Profiles are evolved freely, and gyro-averaging operators are not parametrized, but are functions of the gyrofluid moment variables. The fluid moment hierarchy is closed by approximating the gyrokinetic distribution function as a finite order Hermite-Laguerre polynomial and by determining closure approximations for terms involving the gyrokinetic gyro-averaging operator. The model exactly conserves the gyrokinetic full-F energy invariant evaluated using the Hermite-Laguerre decomposition. The model is suited for qualitative studies of the interplay between turbulence, flows, and dynamically evolving profiles in magnetically confined plasmas. [http://dx.doi.org/10.1063/1.4813241]
\end{abstract}

\section{INTRODUCTION}

Gyrofluid models and fluid models, in general, are widely applied in studies of basic plasma phenomena. The reduced dimensionality compared with more precise kinetic models provides much less computationally expensive tools to advance the understanding of plasma turbulence and the associated transport.

Non-linear simulations of the edge and scrape-off-layer (SOL) regions in magnetically confined fusion plasmas are particularly numerically demanding. These regions are characterized by fluctuation amplitudes that approach or even exceed unity. This especially holds true in the SOL which is dominated by intermittent transport $^{1}$ mainly carried by coherent structures created in the vicinity of the last closed flux surface and expelled into the empty SOL. The filament gradient length scale ${ }^{2,3}$ is typical on the order of $5-20 \rho_{s}$, where $\rho_{s}=\Omega \sqrt{T_{e} / m_{i}}$ is the cold ion acoustic gyroradius; $\Omega$ is the gyro-frequency, $T_{e}$ is the electron temperature, and $m_{i}$ is the ion mass. Temperature measurements in the edge and SOL regions indicate ${ }^{4-9}$ that the ion temperature is comparable to or higher than the electron temperature. It is, therefore, expected that finite Larmor radius (FLR) effects are of importance to the turbulent transport. ${ }^{10}$ In most situations, a stationary equilibrium is never reached. Profiles are formed by the interplay between flows, magnetic topology, turbulence, and the conditions at the plasma edge. The characteristic time-scale for the evolution of profiles is in low-confinement (L-mode) operation an order of magnitude longer than the turbulence de-correlation time, but the two time-scales are comparable in the low to high (L-H) confinement transition and in edge-localized mode (ELM) events when large turbulent structures are expelled into the SOL. Furthermore, the characteristic gradient length-scale of the background profiles in the edge region in H-mode operation becomes comparable with the poloidal gyroradius. ${ }^{11}$ Simulations of the edge and SOL region plasmas therefore require long time-series due to the disparate, but equally

\footnotetext{
${ }^{\text {a)} E l e c t r o n i c ~ m a i l: ~ j m a d @ f y s i k . d t u . d k ~}$
}

important, time-scales, high resolution due to steep gradients and large fluctuation amplitudes, inclusion of FLR effects, self-consistent evolution of the background profiles, and finally the usage fully non-linear models.

Previous gyrofluid models ${ }^{12-16}$ were all partly linearized by splitting fluid fields into small-amplitude fluctuation and stationary background parts. The models were based on the partly linearized delta- $\mathrm{F}$ version of the gyrokinetic model. Essentially, only the $\boldsymbol{E} \times \boldsymbol{B}$-advection non-linearity was kept. In the Maxwell's equations, polarization and magnetization effects were linearized, parallel advection was likewise linearized, and gyro-averages were everywhere evaluated using a fixed background thermal gyro-radius. Previous gyrofluid models are therefore not well-suited for studying edge/SOL turbulence.

In this paper, we present a fully non-linear electromagnetic gyrofluid model consisting of continuity equations for the six first gyrofluid moments, a quasi-neutrality constraint, and the component of Ampere's law parallel to the stationary background magnetic field governing the perturbed perpendicular magnetic field. The gyrofluid model is derived from the so-called full-F gyrokinetic model which is characterized by not splitting the distribution function into background and perturbed parts. The gyrokinetic Maxwell's equations are made tractable by taking terms associated with polarization and magnetization in the long wave-length (LWL) limit. All approximations are made at the gyrokinetic level. This includes the quasi-neutrality assumption and the neglect of parallel magnetization currents in Ampere's law. The gyrofluid continuity equations are obtained by approximating the gyrokinetic distribution function as a finite order HermiteLaguerre polynomial. A closure approximation for the zeroth order moment of the gyro-averaging operator $\Gamma_{1}$ is found by evaluating the Bessel function representation of the kinetic gyro-averaging operator using the decomposed distribution function. Closure approximations for higher order gyrofluid moments of the gyro-averaging operator are given as linear combinations of $\Gamma_{1}$ and the FLR correction $\Gamma_{2}$ to $\Gamma_{1}$, which match to order $k_{\perp}^{2} \rho^{2}$. The gyrofluid model satisfies an exact energy conservation law. The energy invariant equals the 
gyrofluid moment of the corresponding full-F gyrokinetic energy invariant. The knowledge of an exact and physically sensible energy invariant is especially important for nonlinear simulations because unintended violation of energy conservation can lead to sources or sinks of free energy.

The remainder of this paper is organized as follows. In Sec. II, the gyrokinetic Vlasov-Maxwell equations including an exact energy conservation law are derived. The HermitteLaguerre decomposition of the gyrokinetic distribution function and the definition of gyrofluid moment variables are given in Sec. III. The gyrofluid continuity and field equations are derived in Secs. III A and III B, respectively. Closure approximations for the gyro-averaging operators are determined in Sec. III C. The exact energy invariant satisfied by the gyrofluid model is given in Sec. III D. Finally, results are summarized in Sec. IV.

\section{GYROKINETIC MODEL}

The focal point of gyrokinetic theory ${ }^{17}$ is low-frequency electromagnetic fluctuations. Gyrokinetic theory provides a self-consistent Vlasov-Maxwell system of equations in which the fast time-scale associated with the fast cyclotron motion is eliminated. In gyrokinetic theory, the elimination of the fast time-scale is accomplished by asymptotically eliminating the cyclotron motion phase coordinate $\theta$ from the phase-space Lagrangian of a charged particle in an electromagnetic field. ${ }^{18}$ The expansion parameter is given by the standard non-linear gyrokinetic ordering: $q \phi / T \sim \omega / \Omega$ $\sim k_{\|} / k_{\perp} \sim \delta B / B \sim \epsilon_{\delta} \ll 1$ and $k_{\perp} \rho_{i} \sim 1$, where $\phi$ denotes the electrostatic potential, $\delta B$ is the fluctuating magnetic field, $T$ is the temperature, $\omega$ is the characteristic fluctuation frequency, $\Omega=q B / m$ is the background gyro-frequency, where $m$ and $q$ denote mass and charge, respectively, and $B$ is the amplitude of the background magnetic field. $k_{\|}$and $k_{\perp}$ are the characteristic parallel and perpendicular fluctuation wave numbers, respectively. The asymptotic elimination of $\theta$ is accompanied by the construction of an invariant $\dot{\mu}=0$, namely, the magnetic-dipole-moment-like coordinate $\mu$. In the process of eliminating $\theta$ from the charged particle Lagrangian at each order of $\epsilon$, generating functions $S_{n}$ describing the purely oscillatory part of the dynamics are determined. The generating functions in combination with the gyrokinetic Lagrangian determine the gyro-center coordinate transformation $(\boldsymbol{x}, m \boldsymbol{v}) \rightarrow \boldsymbol{Z}=\left(\boldsymbol{X}, v_{\|}, \mu, \theta\right)$.

Using the one-particle gyrokinetic Lagrangian, a gyrokinetic Vlasov-Maxwell action ${ }^{19-22} \mathcal{S}$ can be formulated which uniquely determines the gyro-center coordinate transformation, the equations of motion $\dot{Z}$, the Vlasov equation, the Maxwell's equations, and conservation laws for phase-space, momentum, and energy. The general gyrokinetic VlasovMaxwell system is always simplified because especially the general gyrokinetic Maxwell's equations are not tractable. It is therefore desirable to carry out all simplification on the gyrokinetic action only. Variations of the simplified $\mathcal{S}$ therefore guarantee inter-equation self-consistency, which is otherwise cumbersome to prove and achieve.

Usually, only terms in the second order part of the gyrokinetic Lagrangian are simplified. The second order terms lead to polarization and magnetization effects in the Maxwell's equations. There are two traditional ways of simplifying the Maxwell's equations, which have different benefits and drawbacks. In the so-called "delta-F" approach, the distribution function is split into a stationary background part and a small perturbed part. In the delta-F polarization and the magnetization densities, the perturbed part of the distribution function is neglected. Only linear terms involving the stationary background part of the distribution function are retained. In this approach, arbitrary wavelengths $k_{\perp} \rho_{0} \sim 1$ are everywhere accounted for. Previous gyrofluid models ${ }^{13-16}$ were based on the delta-F gyrokinetic equations.

Here, we derive a gyrofluid model based on the so-called "full-F" 23 gyrokinetic Vlasov-Maxwell equations. In the full-F approach, all terms in the gyrokinetic Lagrangian density which are quadratic or higher in the electromagnetic potentials are taken in the LWL $k_{\perp}^{3} \rho_{0}^{3} \ll 1$. This approximation implies that the corresponding polarization and magnetization densities entering Maxwell's equations appear in the LWL. Arbitrary wavelengths are retained in all terms linear in the electromagnetic potentials, e.g., in the gyro-averaged $\boldsymbol{E} \times \boldsymbol{B}$-drift. Contrary to the delta-F approach, no a priori assumptions are made about $F$. A simultaneous self-consistent treatment of turbulence and equilibrium is therefore possible in the full-F approach. We note that as a consequence of the gyrokinetic ordering $q \phi / T \sim \epsilon_{\delta}$, the gyrokinetic model presented here is only valid for subsonic $\boldsymbol{E} \times \boldsymbol{B}$-flows.

We use a mixed Eulerian-Lagrangian ${ }^{19-22}$ action

$$
\mathcal{S}=\int_{t_{1}}^{t_{2}} d t\left\{\sum_{\alpha} L_{p \alpha}+\int_{-\infty}^{\infty} d^{3} r \mathcal{L}_{f}\right\}
$$

to derive the full-F gyrokinetic Vlasov-Maxwell equations. The particle Lagrangian is given as

$$
L_{p \alpha}=\int d^{6} \boldsymbol{Z}_{0} B_{\| 0}^{*} F_{0 \alpha}\left(\boldsymbol{Z}_{0}\right) \mathcal{L}_{\alpha}\left(Z_{\alpha}\left(\boldsymbol{Z}_{0}, t\right), \phi, A_{\|}\right),
$$

which describes a smooth continuum of particle trajectories $\boldsymbol{Z}_{\alpha}\left(\boldsymbol{Z}_{0}, t\right)$ parametrized by $t ; \alpha$ denotes species, and $B_{\|}^{*}$ is the gyrokinetic volume-element. The particles are labeled by their initial conditions $\left(\boldsymbol{Z}_{0}, t_{0}\right)$. The density of initial conditions is given by a smooth labeling function $F_{0 \alpha}\left(\boldsymbol{Z}_{0}, t_{0}\right)$. The gyrokinetic particle Lagrangian density is taken in the symplectic formalism where the magnetic perturbation $\left\langle A_{\|}\right\rangle$does not enter the Hamiltonian, and which leads to a numerically tractable gyrofluid Ampere's law ${ }^{24}$

$$
\mathcal{L}_{\alpha}=q_{\alpha}\left(\boldsymbol{A}+\left\langle A_{\|}\right\rangle \hat{\boldsymbol{b}}+\frac{m_{\alpha}}{q_{\alpha}} v_{\| \alpha} \hat{\boldsymbol{b}}\right) \cdot \dot{\boldsymbol{X}}_{\alpha}+\frac{m_{\alpha}}{q_{\alpha}} \mu_{\alpha} \dot{\theta}_{\alpha}-H_{\alpha}
$$

where $q_{\alpha}$ and $m_{\alpha}$ denote particle charge and mass, and $\hat{\boldsymbol{b}}=\boldsymbol{B} / B$ is a unit vector directed along the background magnetic field $\boldsymbol{B}=\nabla \times \boldsymbol{A}$. The corresponding volume-element is given as $B_{\|}^{*}=\hat{\boldsymbol{b}} \cdot \boldsymbol{B}^{*}=B+\left[\frac{m_{\alpha}}{q_{\alpha}} v_{\|}+\left\langle A_{\|}\right\rangle\right] \hat{\boldsymbol{b}} \cdot \nabla \times \hat{\boldsymbol{b}}$, where the generalized magnetic field is defined as $\boldsymbol{B}^{*}=\boldsymbol{B}$ $+\frac{m_{\alpha}}{q_{\alpha}} v_{\|} \nabla \times \hat{\boldsymbol{b}}+\nabla \times\left(\left\langle A_{\|}\right\rangle \hat{\boldsymbol{b}}\right)$. The Hamiltonian is given as

$$
H_{\alpha}=\mu_{\alpha} B+\frac{1}{2} m_{\alpha} v_{\|_{\alpha}}^{2}+q_{\alpha} \psi
$$


where we have defined the generalized electric potential

$$
\psi=\langle\phi\rangle-\frac{m_{\alpha} u_{E}^{2}}{2 q_{\alpha}} .
$$

The gyro-averaged electric potential is defined as

$$
\langle\phi\rangle=\frac{1}{2 \pi} \int_{0}^{2 \pi} d \theta \phi\left(\boldsymbol{X}_{\alpha}+\boldsymbol{\rho}_{0 \alpha}\right)
$$

where $\boldsymbol{\rho}_{0 \alpha}=\Omega_{\alpha}^{-1} \hat{\boldsymbol{b}} \times \boldsymbol{v}_{\perp \alpha}$ is the lowest order gyroradius vector and $\boldsymbol{u}_{E}=B^{-1} \hat{\boldsymbol{b}} \times \nabla \phi\left(\boldsymbol{X}_{\alpha}, t\right)$ is the $\boldsymbol{E} \times \boldsymbol{B}$-drift. The electromagnetic field energy Lagrangian density is here given as

$$
\mathcal{L}_{f}=-\frac{\left|\nabla_{\perp} A_{\|}\right|^{2}}{2 \mu_{0}}
$$

where $\mu_{0}$ is the vacuum permeability and the perpendicular part of the gradient operator is defined as $\nabla_{\perp}=-\hat{\boldsymbol{b}} \times(\hat{\boldsymbol{b}} \times \nabla)$. In this article, we assume that the plasma is quasi-neutral, and hence we do not include the electric field energy in $\mathcal{L}_{f}{ }^{25}$ The gyrokinetic Lagrangian Eq. (3) has been simplified in the following ways: (I) all terms of order $k_{\perp}^{3} \rho_{0}^{3}$ or higher in the second order part of the general gyrokinetic Hamiltonian have been neglected. Therefore, only the $\boldsymbol{E} \times \boldsymbol{B}$-energy contained in $\psi$, which is responsible for the important polarization effects, is retained. (II) Shear Alfvénic fluctuations are included through the magnetic perturbation $A_{\|}$, but compressional Alfvén waves are not. (III) All terms non-linear in the magnetic vector potential have been neglected. This approximation implies that the magnetization current density is not present in Ampere's law. (IV) Higher order terms due to inhomogeneities of the background magnetic field have been neglected.

The gyrokinetic distribution function written in terms of Eulerian coordinates $\boldsymbol{Z}$ (Refs. 19 and 22) is defined as

$$
B_{\|}^{*} F_{\alpha}(\boldsymbol{Z}, t)=\int d^{6} \boldsymbol{Z}_{0 \alpha} B_{\| 0}^{*} F_{\alpha 0} \delta^{(6)}\left(\boldsymbol{Z}-\boldsymbol{Z}_{\alpha}\right),
$$

which can be shown to be gyro-angle independent; ${ }^{26} \delta^{(6)}(\boldsymbol{Z})$ is the Dirac delta function. The time-evolution is governed by the gyrokinetic Vlasov equation

$$
\frac{\partial}{\partial t}\left(B_{\|}^{*} F\right)+\nabla \cdot\left(\dot{\boldsymbol{X}} B_{\|}^{*} F\right)+\frac{\partial}{\partial v_{\|}}\left(\dot{v}_{\|} B_{\|}^{*} F\right)=0 .
$$

Species labels are omitted in the remainder of the paper.

In the equations of motion $\dot{\boldsymbol{X}}$ and $\dot{v}_{\|}$, the ratio of the generalized magnetic field $\boldsymbol{B}^{*}$ to the volume-element $B_{\|}^{*}$ enters. In order to avoid ratios of the gyrokinetic velocity-like variables $\left(v_{\|}, \mu\right)$ in the forthcoming derivation of the gyrofluid model, we take

$$
\frac{\boldsymbol{B}^{*}}{B_{\|}^{*}}=\hat{\boldsymbol{b}}+\frac{v_{\|}}{\Omega}(\nabla \times \hat{\boldsymbol{b}})_{\perp}+\frac{\nabla\left\langle A_{\|}\right\rangle \times \hat{\boldsymbol{b}}}{B},
$$

where we neglect the second order contribution $\left\langle A_{\|}\right\rangle \nabla \times \hat{\boldsymbol{b}}$ for the sake of simplicity. Using this expansion, a variation of $S$ with respect to $\boldsymbol{X}_{\alpha}$ gives the gyro-center velocity

$$
\begin{aligned}
\dot{\boldsymbol{X}}= & \hat{\boldsymbol{b}} v_{\|}+\frac{v_{\|}^{2}}{\Omega}(\nabla \times \hat{\boldsymbol{b}})_{\perp}+\frac{\mu \hat{\boldsymbol{b}} \times \nabla \ln B}{q}+\frac{\hat{\boldsymbol{b}} \times \nabla \psi}{B} \\
& +\frac{v_{\|} \nabla\left\langle A_{\|}\right\rangle \times \hat{\boldsymbol{b}}}{B}
\end{aligned}
$$

and a variation of $S$ with respect to $v_{\| \alpha}$ gives the parallel gyro-center acceleration

$$
\begin{aligned}
\dot{v}_{\|}= & -\frac{1}{m}\left[\mu B \hat{\boldsymbol{b}}+\frac{v_{\|} \mu B}{\Omega}(\nabla \times \hat{\boldsymbol{b}})_{\perp}+\frac{\mu B}{B} \nabla\left\langle A_{\|}\right\rangle \times \hat{\boldsymbol{b}}\right] \cdot \nabla \ln B \\
& -\frac{q}{m} \partial_{t}\left\langle A_{\|}\right\rangle-\frac{q}{m}\left[\hat{\boldsymbol{b}}+\frac{v_{\|}}{\Omega}(\nabla \times \hat{\boldsymbol{b}})_{\perp}+\frac{\nabla\left\langle A_{\|}\right\rangle \times \hat{\boldsymbol{b}}}{B}\right] \cdot \nabla \psi .
\end{aligned}
$$

Variation of $S$ with respect to $\phi(\boldsymbol{r})$ yields the quasineutrality constraint

$$
\begin{aligned}
& \sum_{\alpha} \int d^{6} \boldsymbol{Z} B_{\|}^{*}\left[q\left\langle F \delta\left(\boldsymbol{r}-\boldsymbol{X}-\boldsymbol{\rho}_{0}\right)\right\rangle+\nabla \cdot\left(F \delta(\boldsymbol{r}-\boldsymbol{X}) \frac{m}{B^{2}} \nabla_{\perp} \phi\right)\right] \\
& \quad=0 .
\end{aligned}
$$

The last term in Eq. (13) is the polarization charge density which originates from $\boldsymbol{E} \times \boldsymbol{B}$-energy term $-m u_{E}^{2} / 2$ in the gyrokinetic Hamiltonian Eq. (4). We note that energetic consistency is guaranteed by everywhere retaining the second order part $-m u_{E}^{2} / 2$ of $\psi$ in the equations of motion $\dot{\boldsymbol{X}}$ and $\dot{v}_{\|}$. In gyrokinetic models, the polarization charge represents the polarization drift. ${ }^{17}$ The LWL approximation of the second order Hamiltonian therefore implies that FLR corrections ${ }^{10}$ to the polarization drift are neglected.

Similarly, variation of $S$ with respect to $A_{\|}$results in the parallel component of Ampere's law

$$
\frac{1}{\mu_{0}} \nabla_{\perp}^{2} A_{\|}+\sum_{\alpha} q \int d^{6} \boldsymbol{Z} B_{\|}^{*} v_{\|}\left\langle F \delta\left(\boldsymbol{r}-\boldsymbol{X}-\boldsymbol{\rho}_{0}\right)\right\rangle=0 .
$$

Again, we emphasize that the magnetization current does not appear. Therefore, no terms quadratic in the parallel magnetic potential appear in the equations of motion (11) and (12) in order to ensure energetic consistency.

The corresponding energy-invariant for the VlasovMaxwell system is

$$
\begin{aligned}
\mathcal{E}= & \int d^{3} \boldsymbol{r}\left[\frac{\left|\nabla_{\perp} A_{\|}\right|^{2}}{2 \mu_{0}}\right. \\
& \left.+\sum_{\alpha} \int d^{6} \boldsymbol{Z} B_{\|}^{*} F \delta(\boldsymbol{r}-\boldsymbol{X})\left(\mu B+\frac{1}{2} m v_{\|}^{2}+\frac{1}{2} m u_{E}^{2}\right)\right],
\end{aligned}
$$

which consists of the magnetic field energy, the perpendicular and parallel thermal energy, and the $\boldsymbol{E} \times \boldsymbol{B}$-energies, respectively. Note that the electric field energy is absent in as a consequence ${ }^{25}$ of replacing Gauss's law by the quasineutrality condition Eq. (13).

\section{GYROFLUID MODEL}

The gyrofluid model rests on the gyrokinetic model. It is obtained by taking gyrofluid moments of the gyrokinetic Vlasov equation (9) and by expressing the quasi-neutrality 
condition Eq. (13) and Ampere's law (14) in terms of these gyrofluid moments. The gyrofluid moments are defined as

$$
\left\|\mu^{l} v_{\|}^{k}\right\|=\int d v_{\|} d \mu d \theta B_{\|}^{*} F \mu^{l} v_{\|}^{k}
$$

Note that the volume-element in previous linearized gyrofluid models was approximated taking $B_{\|}^{*} \simeq B$. In this article, we derive equations governing the time evolution of the first six gyrofluid moments

$$
\begin{aligned}
N & =\|1\|, & U & =\left\|v_{\|}\right\| / N, \\
P_{\|} & =\left\|m \tilde{v}_{\|}^{2}\right\|, & P_{\perp} & =\|\mu B\|, \\
& =\left\|\mu B \tilde{v}_{\|}\right\|, & Q_{\|,\|} & =\left\|m \tilde{v}_{\|}^{3}\right\|,
\end{aligned}
$$

where $\tilde{v}_{\|}=v_{\|}-U$. In order to close the fluid hierarchy and guarantee consistency, we express the gyrokinetic distribution function as a finite dimensional Hermite-Laguerre polynomial $^{27,28}$ in $\left(v_{\|}, \mu\right)$ space

$$
F=F_{M}(1+\xi),
$$

where

$$
F_{M}=N \frac{1}{2 \pi T_{\perp} m} \sqrt{\frac{1}{2 \pi T_{\|} m}} \exp \left(-\frac{m\left(v_{\|}-U\right)^{2}}{2 T_{\|}}-\frac{\mu B}{T_{\perp}}\right)
$$

is a shifted gyrokinetic Maxwellian and

$$
\begin{aligned}
\xi= & \frac{m Q_{\|, \perp}}{N T_{\perp} T_{\|}}\left(\frac{\mu B}{T_{\perp}}-1\right)\left(v_{\|}-U\right)+\frac{Q_{\|,\|}}{6 N} \sqrt{\frac{m^{3}}{T_{\|}^{3}}} \frac{\tilde{v_{\|}}}{\sqrt{T_{\|} / m}} \\
& \times\left(\frac{\tilde{v}_{\|}^{2}}{T_{\|} / m}-3\right) .
\end{aligned}
$$

The gyrofluid temperatures are defined as $T_{\perp}=P_{\perp} / N$ and $T_{\|}=P_{\|} / N$. Higher order moments entering the gyrofluid moments equations are evaluated using the HermiteLaguerre decomposition given in Eq. (18)

$$
\begin{aligned}
R_{\perp, \perp} & =\left\|(\mu B)^{2}\right\|=2 P_{\perp} T_{\perp}, \quad R_{\perp, \|}=\left\|\mu B m \tilde{v}_{\|}^{2}\right\|=P_{\|} T_{\perp}, \\
R_{\|,\|} & =\left\|\left(m \tilde{v}_{\|}^{2}\right)^{2}\right\|=3 P_{\|} T_{\|}, \quad S_{\perp,\|,\|}=\left\|\mu B m \tilde{v}_{\|}^{3}\right\|=3 Q_{\|, \perp} T_{\|}+Q_{\|,\|} T_{\perp}, \\
S_{\perp, \perp, \|} & =\left\|(\mu B)^{2} \tilde{v}_{\|}\right\|=4 Q_{\perp, \|} T_{\perp}, \quad S_{\|}=\left\|m^{2} \tilde{v}_{\|}^{5}\right\|=10 Q_{\|,\|} T_{\|} .
\end{aligned}
$$

\section{A. Moment equations}

The gyrofluid moment equations are obtained by taking integrals of the form $\int d \mu d v_{\|} d \theta \mu^{l} v_{\|}^{k}$ of the gyrokinetic Vlasov equation (9) using the Hermite-Laguerre decomposition Eq. (18) of the gyrokinetic distribution function $F$

$$
\begin{aligned}
& \frac{\partial}{\partial t} N+\nabla \cdot(\hat{\boldsymbol{b}} U N)+\nabla \cdot\left(\frac{P_{\|}+m N U^{2}}{q B}(\nabla \times \hat{\boldsymbol{b}})_{\perp}\right)+\nabla \cdot\left(\frac{P_{\perp} \hat{\boldsymbol{b}} \times \nabla \ln B}{q B}\right) \\
& +\nabla \cdot\left\|\frac{\hat{\boldsymbol{b}} \times \nabla \psi}{B}\right\|+\nabla \cdot\left\|\frac{v_{\|} \nabla\left\langle A_{\|}\right\rangle \times \hat{\boldsymbol{b}}}{B}\right\|=\Lambda_{N}, \\
& \frac{\partial}{\partial t}(m N U)+\nabla \cdot\left(\hat{\boldsymbol{b}}\left[P_{\|}+m N U^{2}\right]\right)+\nabla \cdot\left(\frac{Q_{\|,\|}+3 U P_{\|}+m N U^{3}}{\Omega}(\nabla \times \hat{\boldsymbol{b}})_{\perp}\right) \\
& +\nabla \cdot\left(\left[U P_{\perp}+Q_{\perp, \|}\right] \frac{\hat{\boldsymbol{b}} \times \nabla \ln B}{\Omega}\right)+\nabla \cdot\left\|m v_{\|} \frac{\hat{\boldsymbol{b}} \times \nabla \psi}{B}\right\|+\nabla \cdot\left\|\frac{m v_{\|}^{2} \nabla\left\langle A_{\|}\right\rangle \times \hat{\boldsymbol{b}}}{B}\right\| \\
& +\left(P_{\perp} \hat{\boldsymbol{b}}+\Omega^{-1}\left[U P_{\perp}+Q_{\perp, \|}\right](\nabla \times \hat{\boldsymbol{b}})_{\perp}+\left\|\frac{\mu B \nabla\left\langle A_{\|}\right\rangle \times \hat{\boldsymbol{b}}}{B}\right\|\right) \cdot \nabla \ln B+q\left\|\partial_{t}\left\langle A_{\|}\right\rangle\right\| \\
& +\|q \hat{\boldsymbol{b}} \cdot \nabla \psi\|+\left\|\frac{m v_{\|}}{B}(\nabla \times \hat{\boldsymbol{b}})_{\perp} \cdot \nabla \psi\right\|+q\left\|\frac{\nabla\left\langle A_{\|}\right\rangle \times \hat{\boldsymbol{b}}}{B} \cdot \nabla \psi\right\|=\Lambda_{U}, \\
& \frac{\partial}{\partial t} P_{\perp}+\nabla \cdot\left(\hat{\boldsymbol{b}}\left[U P_{\perp}+Q_{\perp, \|}\right]\right)+\nabla \cdot\left(\frac{U^{2} P_{\perp}+2 U Q_{\|, \perp}+m^{-1} R_{\perp, \|}}{\Omega}(\nabla \times \hat{\boldsymbol{b}})_{\perp}\right) \\
& +\nabla \cdot\left(R_{\perp, \perp} \frac{\hat{\boldsymbol{b}} \times \nabla \ln B}{q B}\right)+\nabla \cdot\left\|\mu B \frac{\hat{\boldsymbol{b}} \times \nabla \psi}{B}\right\|+\nabla \cdot\left\|\frac{v_{\|} \mu B \nabla\left\langle A_{\|}\right\rangle \times \hat{\boldsymbol{b}}}{B}\right\| \\
& -\left(\left[P_{\perp} U+Q_{\perp, \|]}\right] \hat{\boldsymbol{b}}+\frac{U^{2} P_{\perp}+2 U Q_{\|, \perp}+m^{-1} R_{\perp, \|}}{\Omega}(\nabla \times \hat{\boldsymbol{b}})_{\perp}\right. \\
& \left.+\left\|\mu B \frac{\hat{\boldsymbol{b}} \times \nabla \psi}{B}\right\|+\left\|\frac{v_{\|} \mu B \nabla\left\langle A_{\|}\right\rangle \times \hat{\boldsymbol{b}}}{B}\right\|\right) \cdot \nabla \ln B=\Lambda_{P_{\perp}},
\end{aligned}
$$




$$
\begin{aligned}
& \frac{\partial}{\partial t}\left(P_{\|}+m N U^{2}\right)+\nabla \cdot\left(\hat{\boldsymbol{b}}\left[m N U^{3}+3 U P_{\|}+Q_{\|,\|}\right]\right)+\nabla \cdot\left(\left[m N U^{4}+6 U^{2} P_{\|}+4 U Q_{\|,\|}+m^{-1} R_{\|,\|}\right] \frac{(\nabla \times \hat{\boldsymbol{b}})_{\perp}}{\Omega}\right) \\
& \quad+\nabla \cdot\left(\left[U^{2} P_{\perp}+m^{-1} R_{\perp, \|}+2 Q_{\perp, \|} U\right] \frac{\hat{\boldsymbol{b}} \times \nabla \ln B}{\Omega}\right)+\nabla \cdot\left\|m v_{\|}^{2} \frac{\hat{\boldsymbol{b}} \times \nabla \psi}{B}\right\|+\nabla \cdot\left\|\frac{m v_{\|}^{3} \nabla\left\langle A_{\|}\right\rangle \times \hat{\boldsymbol{b}}}{B}\right\| \\
& \quad+2\left(\left[P_{\perp} U+Q_{\perp, \|}\right] \hat{\boldsymbol{b}}+\Omega^{-1}\left[U^{2} P_{\perp}+2 U Q_{\|, \perp}+m^{-1} R_{\|, \perp}\right](\nabla \times \hat{\boldsymbol{b}})_{\perp}+\left\|\frac{v_{\|} \mu B \nabla\left\langle A_{\|}\right\rangle \times \hat{\boldsymbol{b}}}{B}\right\|\right) \cdot \nabla \ln B \\
& \quad+2 q\left\|v_{\|} \partial_{t}\left\langle A_{\|}\right\rangle\right\|+2 q\left\|v_{\|} \hat{\boldsymbol{b}} \cdot \nabla \psi\right\|+2\left\|\frac{m v_{\|}^{2}}{B}(\nabla \times \hat{\boldsymbol{b}})_{\perp} \cdot \nabla \psi\right\|+2 q\left\|\frac{v_{\|} \nabla\left\langle A_{\|}\right\rangle \times \hat{\boldsymbol{b}}}{B} \cdot \nabla \psi\right\|=\Lambda_{P_{\|}},
\end{aligned}
$$

$$
\begin{aligned}
& \frac{\partial}{\partial t}\left(\frac{Q_{\|, \perp}+U P_{\perp}}{B}\right)+\nabla \cdot\left(\frac{\hat{\boldsymbol{b}}}{B}\left[U^{2} P_{\perp}+m^{-1} R_{\|, \perp}+2 U Q_{\perp, \|}\right]\right) \\
& +\nabla \cdot\left(\left[m U^{3} P_{\perp}+3 U R_{\perp, \|}+3 P_{\|} Q_{\perp, \|}+S_{\perp,\|,\|]} \frac{(\nabla \times \hat{\boldsymbol{b}})_{\perp}}{q B^{2}}\right)+\nabla \cdot\left(\frac{U R_{\perp, \perp}+S_{\|, \perp, \perp}}{q B^{2}} \hat{\boldsymbol{b}} \times \nabla \ln B\right)\right. \\
& +\nabla \cdot\left\|\mu v_{\|} \frac{\hat{\boldsymbol{b}} \times \nabla \psi}{B}\right\|+\nabla \cdot\left\|\frac{\mu B v_{\|}^{2} \nabla\left\langle A_{\|}\right\rangle \times \hat{\boldsymbol{b}}}{B^{2}}\right\| \\
& +\left(\frac{R_{\perp, \perp}}{m B} \hat{\boldsymbol{b}}+\frac{U R_{\perp, \perp}+S_{\|, \perp, \perp}}{m \Omega B}(\nabla \times \hat{\boldsymbol{b}})_{\perp}+\left\|m^{-1}(\mu B)^{2} \nabla\left\langle A_{\|}\right\rangle \times \hat{\boldsymbol{b}}\right\|\right) \cdot \nabla \ln B+\left\|q m^{-1} \mu \partial_{t}\left\langle A_{\|}\right\rangle\right\| \\
& +\frac{q}{m}\|\mu \hat{\boldsymbol{b}} \cdot \nabla \psi\|+\left\|\frac{\mu B v_{\|}}{B^{2}}(\nabla \times \hat{\boldsymbol{b}})_{\perp} \cdot \nabla \psi\right\|+\frac{q}{m}\left\|\mu \frac{\nabla\left\langle A_{\|}\right\rangle \times \hat{\boldsymbol{b}}}{B} \cdot \nabla \psi\right\|=\Lambda_{Q_{\|, \perp}}, \\
& \frac{\partial}{\partial t}\left(Q_{\|,\|}+m N U^{3}+3 U P_{\|}\right)+\nabla \cdot\left(\hat{\boldsymbol{b}}\left[m N U^{4}+\frac{R_{\|,\|}}{m}+6 U^{2} P_{\|}+4 U Q_{\|,\|}\right]\right) \\
& +\nabla \cdot\left(\left[m N U^{5}+\frac{S_{\|}}{m}+\frac{\hat{\boldsymbol{b}} U R_{\|,\|}}{m}+10 U^{2} Q_{\|,\|}+10 P_{\|} U^{3}\right] \frac{(\nabla \times \hat{\boldsymbol{b}})_{\perp}}{\Omega}\right) \\
& +\nabla \cdot\left(\left[S_{\perp,\|,\|}+m U^{2} P_{\perp}+3 m U^{2} Q_{\|, \perp}+3 U R_{\perp, \|}\right] \frac{\hat{\boldsymbol{b}} \times \nabla \ln B}{q B}\right) \\
& +\nabla \cdot\left\|\frac{m v_{\|}^{3} \hat{\boldsymbol{b}} \times \nabla \psi}{B}\right\|+\nabla \cdot\left\|\frac{m v_{\|}^{4} \nabla\left\langle A_{\|}\right\rangle \times \hat{\boldsymbol{b}}}{B}\right\| \\
& +3\left(\left[U^{2} P_{\perp}+\frac{R_{\perp, \|}}{m}+2 U Q_{\perp, \|}\right] \hat{\boldsymbol{b}}+\Omega^{-1}\left[U^{3} P_{\perp}+3 U^{2} Q_{\perp, \|}+\frac{3 U R_{\perp, \|}}{m}+\frac{S_{\|,\|, \perp}}{m}\right](\nabla \times \hat{\boldsymbol{b}})_{\perp}\right) \cdot \nabla \ln B \\
& +3\left\|v_{\|}^{2} \mu \nabla\left\langle A_{\|}\right\rangle \times \nabla \hat{\boldsymbol{b}}\right\| \cdot \nabla \ln B+3 q\left\|v_{\|}^{2} \partial_{t}\left\langle A_{\|}\right\rangle\right\| \\
& +3 q\left\|v_{\|}^{2} \hat{\boldsymbol{b}} \cdot \nabla \psi\right\|+3\left\|m v_{\|}^{3} B^{-1}(\nabla \times \hat{\boldsymbol{b}}) \cdot \nabla \psi\right\|+3 q\left\|v_{\|}^{2} B^{-1} \nabla\left\langle A_{\|}\right\rangle \times \hat{\boldsymbol{b}} \cdot \nabla \psi\right\|=\Lambda_{Q_{\|,\|}} .
\end{aligned}
$$

Equations (23), (25)-(27) govern the time evolution of combinations of the gyrofluid moments defined in Eq. (17). Equations governing the individual gyrofluid moments can be obtained by inserting the lower order gyrofluid moments, e.g., inserting the gyro-center density continuity equation (22) into the parallel gyro-center momentum continuity equation (23).

Dissipative mechanisms are not explicitly added to the moment equations in this work but are simply represented by the " $\Lambda$ " terms on the right hand sides of Eqs. (22)-(27). In previous local gyrofluid models, terms modeling kinetic collisionless dissipation mechanisms, such as Landau damping, non-linear FLR phase mixing, and grad- $B$ and curvature drift phase-mixing are added ${ }^{14,29}$ to the moment equations in a way which mimic the linear response of the kinetic plasma dispersion function. Different sets of closure coefficients were used close to and away from stability threshold in order to mimic the linear kinetic response satisfactorily. Since full$\mathrm{F}$ gyrokinetic models and the global gyrofluid model are fully non-linear, the linear methods used in previous local gyrofluid models are not applicable here. Collisional effects have also been added to local gyrofluid models in an ad-hoc manner. ${ }^{14,16} \mathrm{~A}$ correct inclusion of collisions in gyrofluid models, local and global, is troubled by the fact that collisions take place between particles and not between gyro-centers. Particle collision operators must be expressed in terms of gyro-center positions and velocities, and an explicit gyroaverage must be carried out. The resulting gyro-averaged 
gyrokinetic collision operator is very complex and cannot readily be simplified without breaking conservation of particles, momentum, and energy. ${ }^{30}$ To the knowledge of the author, no gyrokinetic collision operator in the full-F approximation has been derived. A collisional closure of the gyrofluid model presented here is therefore left for future work. The " $\Lambda$ " terms can also represent sinks, sources, and terms added when solving the model equations numerically. The $\Lambda$ 's are kept in order to show how they enter the conserved energy given in Sec. III D, which is not only important for the physical understanding of the model, but is also a good indicator of accuracy in numerical simulations.

All terms involving the gyro-averaging operator are left unspecified. Closure approximations for these terms are discussed and derived in Sec. III C.

\section{B. Field equations}

The quasi-neutrality constraint (13) and Ampere's law (14) are evaluated using the Hermite-Laguerre decomposition Eq. (18). Evaluating the gyro-center integrals using the Dirac delta functions, we get

$$
\begin{gathered}
\sum_{\alpha} q\left\|\frac{\left\langle F\left(\boldsymbol{r}-\boldsymbol{\rho}_{\mathbf{0}}\right)\right\rangle}{F}\right\|+\nabla \cdot\left(\frac{m N}{B^{2}} \nabla_{\perp} \phi\right)=0, \\
-\mu_{0}^{-1} \nabla_{\perp}^{2} A_{\|}=\sum_{\alpha} q\left\|\frac{v_{\|}\left\langle F\left(\boldsymbol{r}-\boldsymbol{\rho}_{\mathbf{0}}\right)\right\rangle}{F}\right\| .
\end{gathered}
$$

In both equations, closure approximations of the terms involving the gyro-averages of the distribution function $F$ are needed. Closure approximations are given in Sec. III C.

\section{Closure approximations for gyro-averaging operators}

In this section, we determine closure approximations for the gyro-averaging operators appearing in the gyrofluid moment equations (22)-(27) and in the field equations (28) and (29). The closure approximations are most easily obtained when gyro-averaged functions are expressed in terms of their inverse Fourier transform

$$
\langle\phi\rangle=\frac{1}{2 \pi} \int_{0}^{2 \pi} d \theta \phi(\boldsymbol{x})=\int d^{3} \boldsymbol{k} e^{i \boldsymbol{k} \cdot \boldsymbol{X}} J_{0}\left(k_{\perp} \rho_{0}\right) \phi_{k}
$$

because the gyro-averaging operator in wave number space is the zeroth order Bessel function of the first kind $J_{0}$. In gyro-center space, $J_{0}$ translates into a linear differential operator. The series expansion of the n'th Bessel function is

$$
J_{n}(z)=\sum_{l=0}^{\infty} \frac{(-1)^{l}}{2^{2 l+n} l !(n+l) !} z^{2 l+n} .
$$

The Bessel function arguments $k_{\perp} \rho_{0}$ depend on the gyrocenter position $\boldsymbol{X}$ and the magnetic-dipole-moment-like coordinate $\mu$ through the amplitude of the zeroth order gyrocenter radius $\rho_{0}=\sqrt{\frac{2 \mu B}{m \Omega^{2}}}$. Moments involving the gyroaveraging operator therefore require an infinite set of moments, and hence closure approximations are required.
Before discussing explicit closure approximations, we note that the effects of the gyro-averages appearing in the gyrokinetic equations of motion Eqs. (11) and (12) and in the gyrokinetic field equations (13) and (14) are different. In the equations of motion, the gyro-averaging operator describes that charged particles, not gyro-centers, interact with the electromagnetic fields. Effectively, the gyroaveraging operator takes particle position-dependent potentials, e.g., $\phi(\boldsymbol{x})$, into gyro-averaged gyro-center-dependent potentials, e.g., $\langle\phi\rangle(\boldsymbol{X}, \mu)$, that interact with gyro-centers. In the field equations, the gyro-averages give the averaged contributions to the charge density at a given position $\boldsymbol{r}$ from gyro-centers whose gyro-orbits intersect $\boldsymbol{r}$. Here, the gyroaveraging operator provides the charge contribution at position $\boldsymbol{r}$ of the gyro-center dependent distribution function $F(\boldsymbol{X})$.

In order to investigate how the two different gyroaveraging operations emerge in gyrofluid models, we evaluate the gyrofluid moment of the gyro-averaged electric potential using the Bessel function series expansion Eq. (31) and the Hermite-Laguerre decomposition Eq. (18)

$$
\left\|\left\langle\phi\left(\boldsymbol{X}+\boldsymbol{\rho}_{0}\right)\right\rangle\right\|=N \Gamma_{1} \phi=N\left(1+\rho^{2} / 2 \nabla_{\perp}^{2}+\cdots\right) \phi,
$$

where $\rho^{2}=T_{\perp} /\left(m \Omega^{2}\right)$. Note that $T_{\perp}$ entering $\Gamma_{1}$ is not fixed at a constant reference temperature as in linearized gyrofluid models. ${ }^{13,16}$ Similarly, the gyrofluid moment of the gyroaveraging operator entering the quasi-neutrality constraint Eq. (13) becomes

$$
\left\|\left\langle F\left(\boldsymbol{r}-\boldsymbol{\rho}_{0}\right)\right\rangle / F\right\|=\left(N+\nabla_{\perp}^{2}\left(N \rho^{2} / 2\right)+\cdots\right),
$$

where we emphasize that $\nabla_{\perp}^{2}$ acts on $\rho$ and $N$ because the distribution function itself is gyro-averaged. Comparing the gyrofluid moments shows that the gyrofluid gyro-averaging operators are Hermitian adjoint, ${ }^{31}$ and hence we can write the gyro-average in Eq. (33) as

$$
\left\|\left\langle F\left(\boldsymbol{r}-\boldsymbol{\rho}_{0}\right)\right\rangle / F\right\|=\Gamma_{1}^{\dagger} N .
$$

We note that in local models, the coefficients in $\Gamma_{1}$ are held constant and $\Gamma_{1}$ is therefore Hermitian. For the parallel current in Ampere's law (14), we get

$$
q\left\|\left\langle v_{\|} F\left(\boldsymbol{r}-\boldsymbol{\rho}_{0}\right)\right\rangle / F\right\|=q\left[\Gamma_{1}^{\dagger} N U+\Gamma_{2}^{\dagger} \frac{Q_{\|, \perp}}{T_{\perp}}\right] .
$$

In the equations of motion Eqs. (11) and (12), the electromagnetic potentials are everywhere operated on by a gradient operator. In order to evaluate gyrofluid moments of these terms, the distribution $F$ and the Bessel function $J_{0}$ must appear together

$$
\|\nabla\langle\phi\rangle\|=N \Gamma_{1} \nabla \phi+\frac{1}{2} \int d^{3} \boldsymbol{k} e^{i \boldsymbol{k} \cdot X}\left\|k_{\perp} \rho_{0} J_{1}\right\| \phi_{k} \nabla \ln B,
$$

where the identity $d J_{0}(z) / d z=-J_{1}(z)$ was used. The second term is evaluated using a trick ${ }^{13}$ 


$$
\left\|k_{\perp} \rho_{0} J_{1}\right\| \approx-\left.\frac{\partial}{\partial \beta}\right|_{\beta=1}\left\|J_{0}\left(k_{\perp} \rho_{0} \beta\right)\right\|=-2 N \Gamma_{2},
$$

where

$$
\Gamma_{2}=T_{\perp} \frac{\partial \Gamma_{1}}{\partial T_{\perp}}=\rho^{2} / 2 \nabla_{\perp}^{2}+\cdots .
$$

$\Gamma_{2}$ is associated with deviations from a circular gyro-orbit due to variations of the perpendicular temperature $T_{\perp}$ and the background magnetic field $B$. The behavior of $\Gamma_{1}$ and $\Gamma_{2}$ in the limit of zero Larmor radius is therefore fundamentally different in the sense that $\Gamma_{1} \rightarrow 1$ for $\rho \rightarrow 0$ whereas $\Gamma_{2} \rightarrow 0$ in the same limit.

Terms involving the gyro-averaged electromagnetic potentials in the higher order moment equations are dealt with in the following way. The gyrofluid moments are evaluated using the Bessel function series expansion Eq. (31) and the distribution function decomposition Eq. (18), e.g.,

$$
\|\mu B\langle\phi\rangle\| \approx P_{\perp}\left(1+\rho^{2} \nabla_{\perp}^{2}+\cdots\right) \phi,
$$

which neither match $\Gamma_{1}$ nor $\Gamma_{2}$. For such terms, we simply combine $\Gamma_{1}$ and $\Gamma_{2}$ such that the series expansion match to order $k_{\perp}^{2}$

$$
\|\mu B\langle\phi\rangle\| \doteq P_{\perp}\left(\Gamma_{1}+\Gamma_{2}\right) \phi .
$$

In order to ease the forthcoming derivation of the conserved energy and to ease comparison with previous gyrofluid equations, we choose to arrange the electromagnetic potentials together with the gyro-averaging operators. As an example, Eq. (36) becomes

$$
\|\nabla\langle\phi\rangle\|=N \nabla\left(\Gamma_{1} \phi\right)+N\left(\Gamma_{2} \phi\right) \nabla \eta,
$$

where

$$
\nabla \eta=\nabla \ln B-\nabla \ln T_{\perp} .
$$

The remaining closure approximations for gyroaveraging terms are

$$
\begin{aligned}
\left\|v_{\|} \nabla\langle\phi\rangle\right\|= & U N \nabla\left(\Gamma_{1} \phi\right)+\Gamma_{2} \phi\left[N U+\frac{Q_{\|, \perp}}{T_{\perp}}\right] \nabla \eta \\
& +\frac{Q_{\|, \perp}}{T_{\perp}} \nabla\left(\Gamma_{2} \phi\right), \\
\|\mu B \nabla\langle\phi\rangle\|= & P_{\perp} \nabla\left(\Gamma_{1} \phi+\Gamma_{2} \phi\right)+2 P_{\perp} \Gamma_{2} \phi \nabla \eta,
\end{aligned}
$$

$$
\begin{aligned}
\left\|v_{\|} \mu B \nabla\langle\phi\rangle\right\|= & U P_{\perp} \nabla\left(\Gamma_{1} \phi+\Gamma_{2} \phi\right)+Q_{\|, \perp} \nabla\left(\Gamma_{1} \phi+3 \Gamma_{2} \phi\right) \\
& +\left(2 U P_{\perp}+4 Q_{\|, \perp}\right) \Gamma_{2} \phi \nabla \eta,
\end{aligned}
$$

$$
\begin{aligned}
\left\|m v_{\|}^{2} \nabla\langle\phi\rangle\right\|= & {\left[P_{\|}+m N U^{2}\right] \nabla\left(\Gamma_{1} \phi\right)+\frac{2 U Q_{\perp, \|}}{T_{\perp}} \nabla\left(\Gamma_{2} \phi\right) } \\
& +\Gamma_{2} \phi\left[m N U^{2}+P_{\|}+\frac{2 U Q_{\perp, \|}}{T_{\perp}}\right] \nabla \eta,
\end{aligned}
$$

Terms involving $\phi$ and $A_{\|}$are evaluated in the same way

$$
\begin{aligned}
\left\|v_{\|} \nabla\left\langle A_{\|}\right\rangle \times \hat{\boldsymbol{b}} \cdot \nabla\langle\phi\rangle\right\|= & N U\left[\nabla\left(\Gamma_{1} A_{\|}\right) \times \hat{\boldsymbol{b}} \cdot \nabla\left(\Gamma_{1} \phi\right)+\Gamma_{2} A_{\|} \nabla \eta \times \hat{\boldsymbol{b}} \cdot \nabla\left(\Gamma_{1} \phi+\Gamma_{2} \phi\right)\right. \\
& \left.+\Gamma_{2} \phi \nabla\left(\Gamma_{1} A_{\|}+\Gamma_{2} A_{\|}\right) \times \hat{\boldsymbol{b}} \cdot \nabla \eta+\nabla\left(\Gamma_{2} A_{\|}\right) \times \hat{\boldsymbol{b}} \cdot \nabla\left(\Gamma_{2} \phi\right)\right] \\
& +\frac{Q_{\|, \perp}}{T_{\perp}}\left[\nabla\left(\Gamma_{1} A_{\|}+2 \Gamma_{2} A_{\|}\right) \times \hat{\boldsymbol{b}} \cdot \nabla\left(\Gamma_{2} \phi\right)+\nabla\left(\Gamma_{2} A_{\|}\right) \times \hat{\boldsymbol{b}} \cdot \nabla\left(\Gamma_{1} \phi\right)\right. \\
& \left.+\Gamma_{2} A_{\|} \nabla \eta \times \hat{\boldsymbol{b}} \cdot \nabla\left(\Gamma_{1} \phi+3 \Gamma_{2} \phi\right)+\Gamma_{2} \phi \nabla\left(\Gamma_{1} A_{\|}+3 \Gamma_{2} A_{\|}\right) \times \hat{\boldsymbol{b}} \cdot \nabla \eta\right],
\end{aligned}
$$




$$
\begin{aligned}
\left\|\nabla\left\langle A_{\|}\right\rangle \times \hat{\boldsymbol{b}} \cdot \nabla\langle\phi\rangle\right\|= & N\left[\nabla\left(\Gamma_{1} A_{\|}\right) \times \hat{\boldsymbol{b}} \cdot \nabla\left(\Gamma_{1} \phi\right)+\Gamma_{2} A_{\|} \nabla \eta \times \hat{\boldsymbol{b}} \cdot \nabla\left(\Gamma_{1} \phi+\Gamma_{2} \phi\right)\right. \\
& \left.+\Gamma_{2} \phi \nabla\left(\Gamma_{1} A_{\|}+\Gamma_{2} A_{\|}\right) \times \hat{\boldsymbol{b}} \cdot \nabla \eta+\nabla\left(\Gamma_{2} A_{\|}\right) \times \hat{\boldsymbol{b}} \cdot \nabla\left(\Gamma_{2} \phi\right)\right], \\
\left\|\mu B \nabla\left\langle A_{\|}\right\rangle \times \hat{\boldsymbol{b}} \cdot \nabla\langle\phi\rangle\right\|=P_{\perp}[ & \nabla\left(\left[\Gamma_{1}+\Gamma_{2}\right] A_{\|}\right) \times \hat{\boldsymbol{b}} \cdot \nabla\left(\left[\Gamma_{1}+\Gamma_{2}\right] \phi\right)+2 \Gamma_{2} A_{\|} \nabla \eta \times \hat{\boldsymbol{b}} \cdot \nabla\left(\Gamma_{1} \phi+2 \Gamma_{2} \phi\right) \\
+ & \left.2 \Gamma_{2} \phi \nabla\left(\Gamma_{1} A_{\|}+2 \Gamma_{2} A_{\|}\right) \times \hat{\boldsymbol{b}} \cdot \nabla \eta+2 \nabla\left(\Gamma_{2} A_{\|}\right) \times \hat{\boldsymbol{b}} \cdot \nabla\left(\Gamma_{2} \phi\right)\right], \\
\left\|m v_{\|}^{2} \nabla\left\langle A_{\|}\right\rangle \times \hat{\boldsymbol{b}} \cdot \nabla\langle\phi\rangle\right\|= & \left(P_{\|}+m N U^{2}\right)\left[\nabla\left(\Gamma_{1} A_{\|}\right) \times \hat{\boldsymbol{b}} \cdot \nabla\left(\Gamma_{1} \phi\right)+\Gamma_{2} A_{\|} \nabla \eta \times \hat{\boldsymbol{b}} \cdot \nabla\left(\Gamma_{1} \phi+\Gamma_{2} \phi\right)\right. \\
& \left.+\Gamma_{2} \phi \nabla\left(\Gamma_{1} A_{\|}+\Gamma_{2} A_{\|}\right) \times \hat{\boldsymbol{b}} \cdot \nabla \eta+\nabla\left(\Gamma_{2} A_{\|}\right) \times \hat{\boldsymbol{b}} \cdot \nabla\left(\Gamma_{2} \phi\right)\right] \\
& +2 \frac{Q_{\|, \perp}}{T_{\perp}}\left[\nabla\left(\Gamma_{1} A_{\|}+2 \Gamma_{2} A_{\|}\right) \times \hat{\boldsymbol{b}} \cdot \nabla\left(\Gamma_{2} \phi\right)+\nabla\left(\Gamma_{2} A_{\|}\right) \times \hat{\boldsymbol{b}} \cdot \nabla\left(\Gamma_{1} \phi\right)\right. \\
& \left.+\Gamma_{2} A_{\|} \nabla \eta \times \hat{\boldsymbol{b}} \cdot \nabla\left(\Gamma_{1} \phi+3 \Gamma_{2} \phi\right)+\Gamma_{2} \phi \nabla\left(\Gamma_{1} A_{\|}+3 \Gamma_{2} A_{\|}\right) \times \hat{\boldsymbol{b}} \cdot \nabla \eta\right] .
\end{aligned}
$$

Note that the terms involving two gyro-averages have not been simplified as in Ref. 15. So far, no explicit choice for the gyro-averaging operators $\Gamma_{1}$ and $\Gamma_{2}$ has been given. One particular choice is to use the Padé approximant

$$
\Gamma_{1}=\frac{1}{1-\rho^{2} / 2 \nabla_{\perp}^{2}},
$$

which is easily implemented in numerical $\operatorname{codes}^{13}$ and is well behaved at large $k_{\perp} \rho$. Using the definition Eq. (38), the Padé approximant for $\Gamma_{2}$ becomes

$$
\Gamma_{2}=\frac{-b / 2}{(1+b / 2)^{2}} .
$$

The moment equations (22)-(27) together with the field equations (28) and (29) form a closed model when the gyroaveraging closure approximations are inserted. The model is based on the full-F gyrokinetic model given in Sec. II. In the delta-F approximation of the gyrokinetic equations, the gyrokinetic distribution function is split into background and perturbed parts, whereas the full distribution function is retained in the full-F approximation. The two approximations otherwise only differ in how polarization and magnetization densities are simplified. In the delta-F approximation, only the background part of the distribution function is retained in the polarization and magnetization terms. The full distribution function is everywhere retained in the full-F approximation, but the polarization and magnetization terms are taken in the LWL limit. The delta-F and the full-F models therefore agree if the polarization and magnetization terms in the delta-F model are taken in the LWL limit, and the distribution function in the full-F model is split into background and perturbed parts, and the perturbed part of the distribution function is discarded in the polarization and magnetization terms. In Ref. 32, it is shown that local delta-F gyrokinetic based gyrofluid models are supersets of local low-frequency drift-fluid models. The gyrofluid gyro-averaging operators $\Gamma_{1}$ and $\Gamma_{2}$ agree in the LWL with the corresponding operators in Ref. 32 when evaluated at constant background temperatures and magnetic field strength. The global gyrofluid model presented here is therefore also a superset of local drift fluid models. We expect that a similar correspondence exists between global drift fluid models ${ }^{33}$ and the global gyrofluid model presented here. However, proving this assertion will require a substantial amount of further work because of the non-linear nature of the field equations, and is therefore left for future work.

\section{Energy conservation}

In this section, we present the conserved energy of the gyrofluid model. The energy conservation law is

$$
\partial_{t} \mathcal{E}=\Lambda .
$$

The energy $\mathcal{E}$ is obtained by inserting the Hermite-Laguerre decomposition Eq. (18) into the gyrokinetic energy conservation law Eq. (15)

$$
\mathcal{E}=\sum_{\alpha} \int d^{3} \boldsymbol{X} \frac{m N u_{E}^{2}}{2}+\frac{m N u^{2}}{2}+\frac{P_{\|}}{2}+P_{\perp}+\frac{\left|\nabla_{\perp} A_{\|}\right|^{2}}{2 \mu_{0}} .
$$

The energy consists of the $\boldsymbol{E} \times \boldsymbol{B}$-energy density, the parallel kinetic energy density, the parallel and perpendicular internal energy densities, and the perturbed magnetic field energy density, respectively. The dissipative effects $\Lambda$ originating from the unspecified dissipative terms on the right hand sides of the gyrofluid moment equations (22)-(27) are given by

$$
\Lambda=\sum_{\alpha} \int d^{3} \boldsymbol{X} q\left(\chi-\Gamma_{2} \phi\right) \Lambda_{N}+\frac{q \Gamma_{2} \phi}{T_{\perp}} \Lambda_{P_{\perp}}+\Lambda_{P_{\perp}}+\frac{\Lambda_{P_{\|}}}{2},
$$

where $\chi=\Gamma_{1} \phi-m u_{E}^{2} / e$ is the gyrofluid generalized electric potential.

The energy conservation law is exactly obeyed by the gyrofluid moment equations (22)-(27) and the gyrofluid field equations (28) and (29) in the combination with the closure approximations given in Sec. IIIC. The energy conservation law Eq. (57) is proved by explicitly evaluating the time 
derivative of $\mathcal{E}$ using the gyrofluid moment equations (22)-(27) and the gyrofluid field equations (28) and (29). The evaluation is mostly straightforward and follows the same path as in local gyrofluid models. ${ }^{34}$ The only term in $\mathcal{E}$ which requires special attention is the $\boldsymbol{E} \times \boldsymbol{B}$-energy part. Multiplying the quasi-neutrality constraint Eq. (28) by the electric potential $\phi$ and performing partial integration, ${ }^{31}$ we get

$$
\frac{d}{d t} \int d^{3} \boldsymbol{X} \frac{m N u_{E}^{2}}{2}=\int d^{3} \boldsymbol{r} q\left[\chi-\left(\Gamma_{2} \phi\right)\right] \frac{\partial N}{\partial t}+q \frac{\left(\Gamma_{2} \phi\right)}{T_{\perp}} \frac{\partial P_{\perp}}{\partial t} .
$$

Terms quadratic in $\phi$ cancel. The remaining terms, except non-specified dissipative terms, are canceled by terms in the internal and parallel kinetic energy terms of $\mathcal{E}$ which depend on $\phi$ and $A_{\|}$. We note that the exact conservation of the energy invariant $\mathcal{E}$ is independent of the choice of $\Gamma_{1}$ provided that $\Gamma_{2}$ is given by Eq. (38).

\section{CONCLUSIONS}

In summary, we have derived a non-linear electromagnetic six-moment gyrofluid model based on the full-F version of gyrokinetics. The time evolution of the gyrofluid moments is governed by Eqs. (22)-(27), the quasi-neutrality constraint is given in Eq. (28), and the parallel component of Ampere's law for the perturbed perpendicular magnetic field is given in Eq. (29). Closure approximations for all terms involving gyroaveraging are found in Eqs. (41) and (43)-(54). No terms in the gyrofluid model have been linearized including gyroaveraging operators, the quasi-neutrality constraint, and Ampere's law. The model is therefore suited for qualitative studies of basic mechanisms such as the interplay between the small scale turbulence, sub-sonic large scale flows, and dynamically evolving profiles. The fluid hierarchy is closed by taking the gyrokinetic distribution function as a finite dimensional Hermite-Laguerre polynomial in the parallel gyro-center velocity and the magnetic-dipole-moment-like coordinate having the gyrofluid moments retained in the model as coefficients. The quasi-neutrality constraint, Ampere's law, and the closure approximations of terms involving the gyro-average are calculated using the decomposed gyrokinetic distribution function. The exactly conserved energy invariant therefore equals the gyrokinetic energy conservation law evaluated with the decomposed gyrokinetic distribution function.

We would like to emphasize that to our knowledge the model presented here is the first published global gyrofluid model. Global fluid models including FLR effects were derived by Strintzi et al. in Refs. 31 and 35. The models were based on a constructed fluid Lagrangian and were derived using a constrained variational principle. These models were also termed gyrofluid models. However, the fluid moment variables in the global model by Strintzi et al. are not gyrofluid moments as defined in Eq. (16), but are standard velocity moments

$$
\|\chi\|_{p}=\int d^{3} \boldsymbol{v} f(\boldsymbol{x}, \boldsymbol{v}) \chi(\boldsymbol{v})
$$

which only equal the gyrofluid moment Eq. (16) to zeroth order on the gyrokinetic smallness parameter $\epsilon_{\delta} .{ }^{10,24,36}$ Therefore, the moment equations in Strintzi's model have non-physical diamagnetic advection terms which are eliminated by hand after performing the constrained variation. Diamagnetic advection terms do not enter gyrofluid models because the gyro-viscous cancellation problem is bypassed ${ }^{24,36}$ using gyrokinetic theory.

\section{ACKNOWLEDGMENTS}

The author would like to thank V. Naulin, A. H. Nielsen, M. Salewski, and J. J. Rasmussen for helpful suggestions and careful reading of the manuscript. This work was supported by an EFDA fusion researcher fellowship (WP11-FRFRISOE/MADSEN).

${ }^{1}$ V. Naulin, J. Nucl. Mater. 363, 24 (2007).

${ }^{2}$ O. E. Garcia, N. H. Bian, and W. Fundamenski, Phys. Plasmas 13, 82309 (2006).

${ }^{3}$ J. A. Boedo, J. Nucl. Mater. 390, 29 (2009).

${ }^{4}$ J. Adamek et al., Contrib. Plasma Phys. 48, 395 (2008).

${ }^{5}$ G. S. Xu, V. Naulin, W. Fundamenski, J. J. Rasmussen, A. H. Nielsen, and B. N. Wan, Phys. Plasmas 17, 22501 (2010).

${ }^{6}$ K. Uehara, T. Kawakami, H. Amemiya, K. Höthker, A. Cosler, and W. Bieger, Nucl. Fusion 38, 1665 (1998).

${ }^{7}$ M. Reich, E. Wolfrum, J. Schweinzer, H. Ehmler, L. D. Horton, J. Neuhauser, and ASDEX Upgrade Team, Plasma Phys. Controlled Fusion 46, 797 (2004).

${ }^{8}$ A. S. Wan, B. Lipschultz, F. S. McDermott, and J. L. Terry, J. Nucl. Mater. 162, 292 (1989).

${ }^{9}$ M. Kočan, J. P. Gunn, S. Carpentier-Chouchana, A. Herrmann, A. Kirk, M. Komm, H. W. Müller, J.-Y. Pascal, R. A. Pitts, V. Rohde, and P. Tamain, J. Nucl. Mater. 415, S1133 (2011).

${ }^{10}$ J. Madsen, O. E. Garcia, J. S. Larsen, V. Naulin, A. H. Nielsen, and J. J. Rasmussen, Phys. Plasmas 18, 112504 (2011).

${ }^{11}$ K. H. Burrell, E. J. Doyle, P. Gohil, R. J. Groebner, J. Kim, R. J. La Haye, L. L. Lao, R. A. Moyer, T. H. Osborne, W. A. Peebles, C. L. Rettig, T. H. Rhodes, and D. M. Thomas, Phys. Plasmas 1, 1536 (1994).

${ }^{12}$ G. Knorr, F. R. Hansen, J. P. Lynov, H. L. Pécseli, and J. J. Rasmussen, Phys. Scr. 38, 829 (1988).

${ }^{13}$ W. Dorland and G. W. Hammett, Phys. Fluids B 5, 812 (1993).

${ }^{14}$ M. A. Beer and G. W. Hammett, Phys. Plasmas 3, 4046 (1996)

${ }^{15}$ P. B. Snyder and G. W. Hammett, Phys. Plasmas 8, 3199 (2001).

${ }^{16}$ B. Scott, Phys. Plasmas 17, 102306 (2010).

${ }^{17}$ A. J. Brizard and T. S. Hahm, Rev. Mod. Phys. 79, 421 (2007).

${ }^{18}$ H. Goldstein, C. Poole, and J. Safko, Classical Mechanics, edited by A. Black (Addison Wesley, San Francisco, 2002).

${ }^{19}$ B. M. Boghosian, Ph.D. dissertation, University of California, Los Angeles, 1987.

${ }^{20}$ H. Sugama, Phys. Plasmas 7, 466 (2000).

${ }^{21}$ A. J. Brizard, Phys. Plasmas 7, 4816 (2000).

${ }^{22}$ J. Madsen, Phys. Plasmas 17, 82107 (2010).

${ }^{23}$ T. S. Hahm, L. Wang, and J. Madsen, Phys. Plasmas 16, 22305 (2009).

${ }^{24}$ A. Brizard, Phys. Fluids B 4, 1213 (1992).

${ }^{25}$ D. C. Restrepo and D. Pfirsch, J. Plasma Phys. 71, 503 (2005).

${ }^{26}$ D. H. E. Dubin, J. A. Krommes, C. Obermann, and W. W. Lee, Phys Fluids 26, 3524 (1983).

${ }^{27}$ R. Balescu, Transport Processes in Plasmas (North-Holland, Amsterdam, 1988), Vol. 1

${ }^{28}$ G. W. Hammett, M. A. Beer, W. Dorland, S. C. Cowley, and S. A. Smith, Plasma Phys. Controlled Fusion 35, 973 (1993).

${ }^{29}$ G. W. Hammett and F. W. Perkins, Phys. Rev. Lett. 64, 3019 (1990).

${ }^{30}$ J. Madsen, Phys. Rev. E 87, 011101 (2013).

${ }^{31}$ D. Strintzi and B. Scott, Phys. Plasmas 11, 5452 (2004).

${ }^{32}$ B. D. Scott, Phys. Plasmas 14, 102318 (2007).

${ }^{33}$ A. Zeiler, J. F. Drake, and B. Rogers, Phys. Plasmas 4, 2134 (1997).

${ }^{34}$ B. D. Scott, Phys. Plasmas 12, 102307 (2005).

${ }^{35}$ D. Strintzi, B. D. Scott, and A. J. Brizard, Phys. Plasmas 12, 52517 (2005).

${ }^{36}$ E. V. Belova, Phys. Plasmas 8, 3936 (2001). 